\title{
Electrical measurement of cross-sectional position of particles flowing through a microchannel
}

\author{
Riccardo Reale $^{1} \cdot$ Adele De Ninno $^{1} \cdot$ Luca Businaro $^{2} \cdot$ Paolo Bisegna $^{1} \cdot$ Federica Caselli ${ }^{1}[$
}

Received: 15 December 2017 / Accepted: 6 March 2018 / Published online: 16 March 2018

(c) The Author(s) 2018

\begin{abstract}
The first high-throughput system for the electrical detection of cross-sectional position and velocity of individual particles flowing through a rectangular microchannel is presented. Lateral position (along channel width) and vertical position (along channel height) are measured using two different sets of coplanar electrodes. In particular, the ratio of travel times measured with electrodes generating a current flow transverse or oblique with respect to particle trajectory yields lateral position. The relative prominence and transit time of a bipolar double-Gaussian signal obtained with a suited electrode configuration, respectively, supply vertical position and velocity. The operating principle is presented by means of finite element numerical simulations. The method is experimentally validated by comparing the electrical estimates of position and velocity of polystyrene beads with optical estimates obtained by processing high-speed images. The system is used to observe bead focusing at different particle Reynolds numbers. This system, providing a fully electrical characterization of single-particle motion, represents a powerful tool, e.g. to understand fluid motion at the microscale, in particle separation studies, or to assess the performance of particle focusing devices. Moreover, it can be simultaneously used to perform single-cell impedance spectroscopy, thus achieving an unprecedented multiparamteric characterization.
\end{abstract}

Keywords Electrical impedance $\cdot$ Microfluidics $\cdot$ Position detection $\cdot$ Coplanar electrodes

\section{Introduction}

Microfluidic applications are continuing to expand in the fields of nanotechnology, manufacturing and life sciences, and therefore, there is a pressing need for methods to characterize fluid behaviour at the microscale and suspended particle motion (Williams et al. 2010; Winer et al. 2014; Tasadduq et al. 2015). As an example, detecting cross-sectional position of flowing particles is essential in the investigation of self-ordering mechanisms (Gao et al. 2017), inertial particle focusing (Di Carlo 2009) or active particle focusing [e.g.

Electronic supplementary material The online version of this article (https://doi.org/10.1007/s10404-018-2055-3) contains supplementary material, which is available to authorized users.

Federica Caselli

caselli@ing.uniroma2.it

1 Department of Civil Engineering and Computer Science, University of Rome Tor Vergata, 00133 Rome, Italy

2 Institute for Photonics and Nanotechnologies, Italian National Research Council, 00156 Rome, Italy dielectrophoretic (Shaker et al. 2014) and acustophoretic (Muller et al. 2013)], which have applications in single-cell analysis and sorting (Wyatt Shields IV et al. 2015). In fact, under specific force fields, position can directly indicate the property of target cells (Wang et al. 2017).

Numerous optical methods have been developed to obtain flow field and particle position in microfluidic channels. Conventional video microscopy depicts particle motion projected onto a plane defined by the focus of the objective. For many applications (e.g. micromixers or particle focusing devices), visualization of motion in the third dimension is required to fully understand the trajectory of particles (Tasadduq et al. 2015). To that aim, multi-camera (e.g. stereoscopic imaging, tomographic imaging) and single camera (e.g. confocal scanning microscopy, anamorphic/astigmatic imaging, digital holographic microscopy, deconvolution and defocusing microscopy) approaches have been proposed [for a description and comparative analysis see e.g. the reviews Lee and Kim (2009), Williams et al. (2010) and Cierpka and Kähler (2012)]. However, those methods require expensive and intricate optical setups involving numerous lenses, aperture designs and light sources, or demand for refined 
and intensive processing of massive image data (Winer et al. 2014; Lin and $\mathrm{Su} 2016$ ).

An attractive alternative to optical systems is represented by electrical impedance-based approaches (Wang et al. 2017; Caselli et al. 2017; Brazey et al. 2018). Impedance-based microfluidic devices have been extensively studied in the last decade due to their simplicity and potential for low-cost and portable implementation. In particular, microfluidic impedance cytometers for label-free analysis of single particles and cells at high throughput (Cheung et al. 2010; Sun and Morgan 2010; Petchakup et al. 2017) have been used in different biological assays, including particle sizing and counting, cell phenotyping and disease diagnostics [e.g. Haandbæk et al. (2016), McGrath et al. (2017), Rohani et al. (2017), Esfandyarpour et al. (2017) and Rollo et al. (2017)]. Those systems use electrodes integrated in a microchannel to measure variation in the electric field caused by the transit of particles. The accuracy of the technique is challenged by the positional dependence issue, i.e. identical particles flowing in the microchannel along different trajectories provide different signals (Spencer and Morgan 2011). This is due to fringing electric field and may introduce blurring in the estimated particle properties. Compensation strategies aimed at solving the positional dependence issue have been proposed by our group, thus achieving high accuracy particle sizing. To this end, new metrics have been devised, correlating with either lateral (i.e. along channel width) (Caselli et al. 2017, 2018) or vertical (i.e. along channel height) (Spencer et al. 2016; De Ninno et al. 2017) particle position.

Those metrics are here exploited for quantitative particle localization. The first impedance-based device for high-throughput determination of cross-sectional (i.e. both lateral and vertical) position and velocity of single particles flowing through a rectangular microchannel is presented. The system comprises two consecutive regions, each equipped with a different set of coplanar electrodes (Fig. 1). As detailed in Sect. 2,

(i) an electrical estimate, $X$, of lateral particle coordinate ( $x$-coordinate) is obtained from the ratio $R$ of the travel times measured using two sets of electrodes placed in lateral channels (first region, Fig. 1b);

(ii) an electrical estimate, $Y$, of vertical particle coordinate ( $y$-coordinate) is obtained from the relative prominence $P$ (De Ninno et al. 2017) of a bipolar double-Gaussian impedance signal recorded using five electrodes spanning the width of the main channel (second region, Fig. 1c);

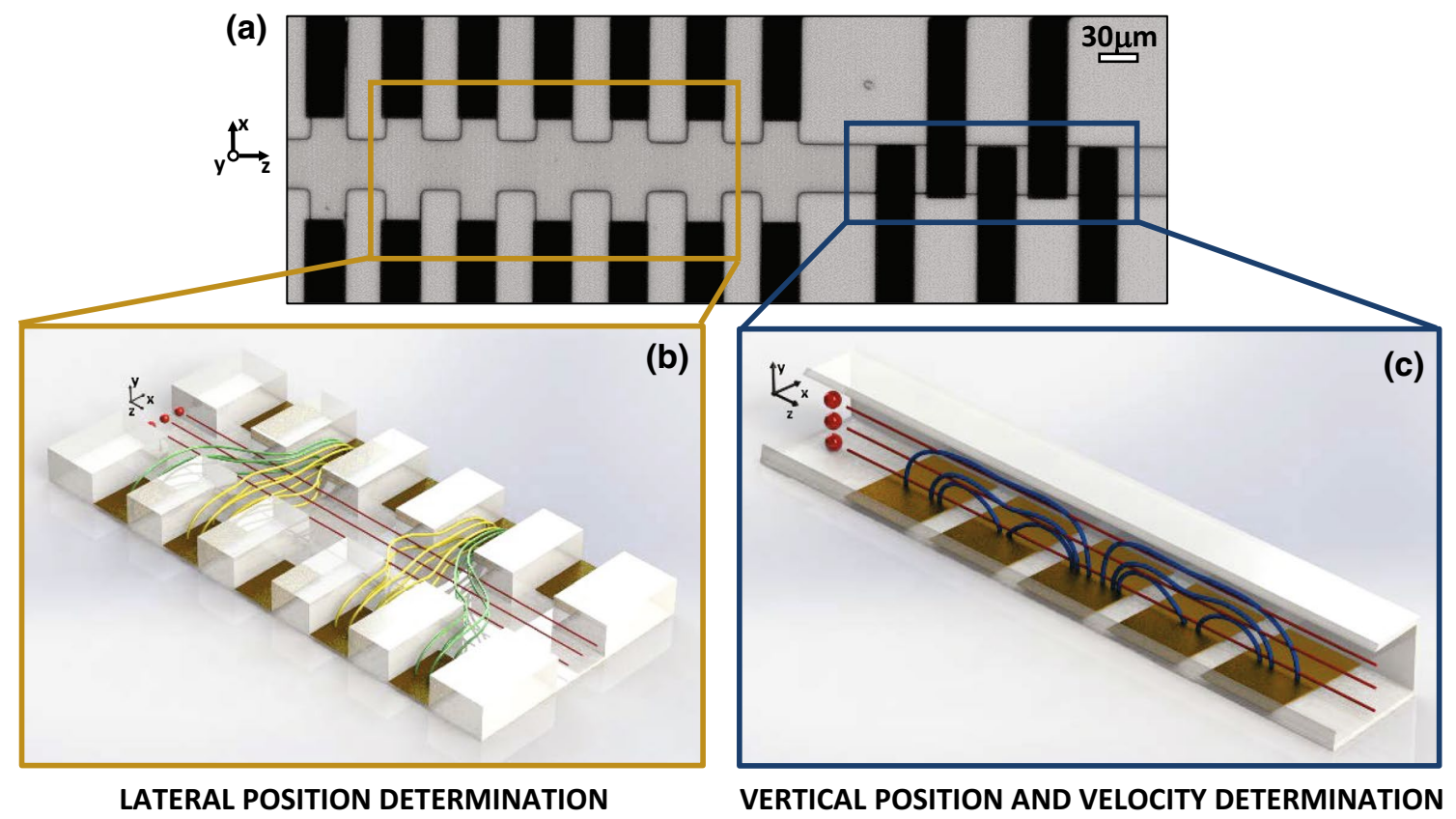

Fig. 1 a Microscope image of the electrical sensing area. The region highlighted with a yellow box, comprising five pairs of lateral electrodes, is used for the determination of particle lateral position. The region highlighted with a blue box, formed by five electrodes spanning the channel width, is used for the determination of particle vertical position and velocity. $\mathbf{b}, \mathbf{c} 3 \mathrm{D}$ rendered models of the two meas-

uring regions. Lateral position is determined by comparing signals relevant to transverse and oblique current flows (yellow and green field lines in panel (b), respectively). Vertical position and velocity are determined exploiting an electrode configuration that generates a peculiar current distribution (blue field lines), characterized by alternating high- and low-field regions (colour figure online) 
(iii) particle velocity is extracted from the latter signal, dividing the spatial length of the sensing zone by the transit time.

A thorough validation of the proposed approach is reported. Impedance signals for 6 - and $10-\mu \mathrm{m}$ polystyrene beads have been collected. The electrical estimates of particle cross-sectional position and velocity are compared with the estimates derived by image processing of optical frames recorded using a high-speed camera. Moreover, the method is used to monitor the bead flow under different flow rates, and partial inertial focusing is observed.

The paper is organized as follows: the operating principle enabling the electrical estimate of particle cross-sectional position and velocity is presented in Sect. 2, the experimental methods are described in Sect. 3, and validation and results are presented in Sect. 4. Finally, conclusions are drawn.

\section{Operating principle}

\subsection{Electrical estimate of particle lateral position}

The chip region used for the electrical estimation of particle lateral position (i.e. $x$-coordinate) exploits the concept of liquid electrodes (Demierre et al. 2007). They are vertical equipotential surfaces generated on apertures of the main channel walls by recessed coplanar electrodes patterned at the bottom of dead-end lateral chambers (Fig. 1b). Liquid electrodes provide a nearly homogeneous electric field over the total channel height while keeping a simple process flow with a single planar metal layer (Mernier et al. 2012).

In the present work, five pairs of coplanar electrodes are considered and the following wiring scheme is proposed (Fig. 2a): an AC voltage is applied to two stimulation electrodes on one side of the main channel (the 2 nd and the 4 th electrodes along $z$, at $x>0$ ), and two differential current signals are measured using four sensing electrodes located on the opposite side (i.e. at $x<0$ ). The first signal, denoted $I_{\mathrm{TSV}}$, is the difference in electric current flowing through the sensing electrodes with the same $z$-coordinate as the stimulation electrodes (the 2 nd and the 4 th electrodes along $z$ ) which features a current path transverse to particle flow. The second signal, denoted $I_{\mathrm{OBQ}}$, is the difference in electric current flowing through two sensing electrodes with a $z$-coordinate different from the stimulating electrodes (the 1 st and the 5th electrodes along $z$ ) featuring a current path oblique with respect to particle flow. An analogous wiring scheme was introduced in Spencer et al. (2016) for a chip comprising five pairs of facing electrodes, aimed at solving the positional dependence issue.
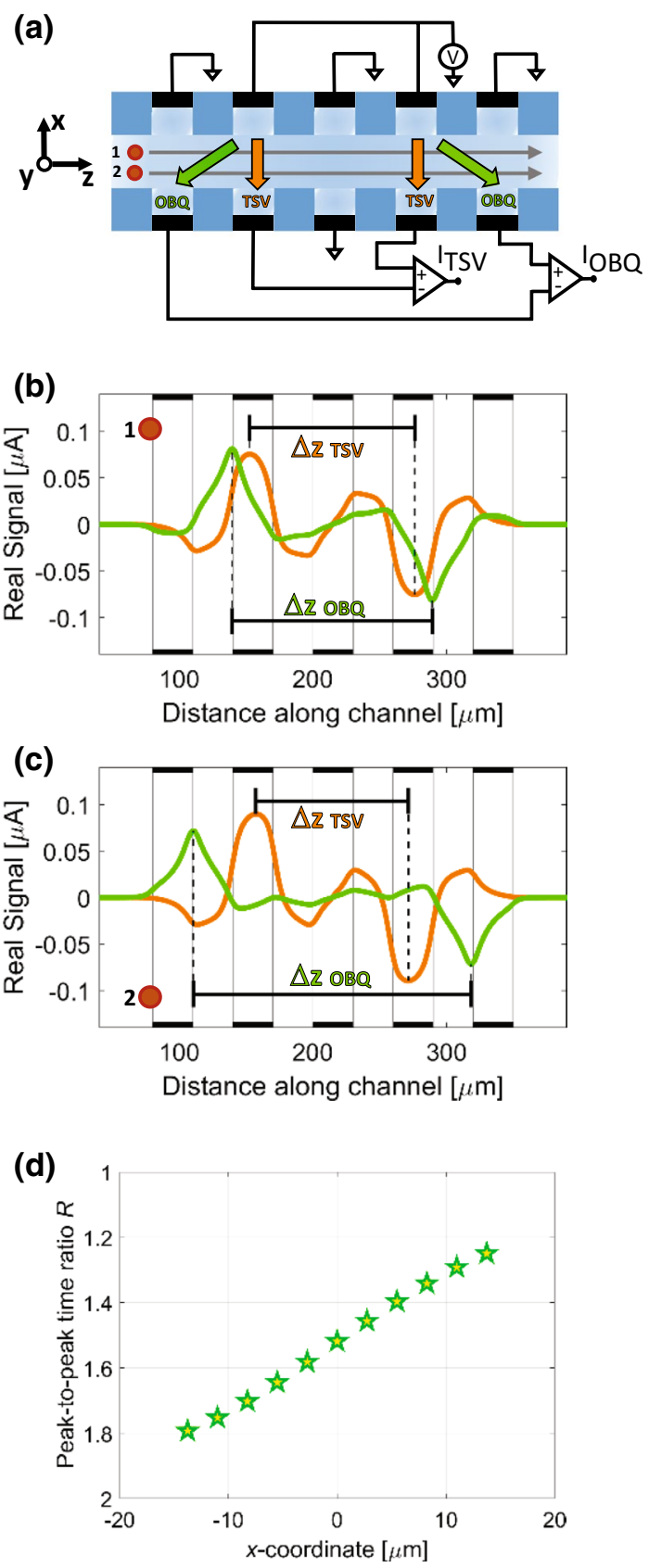

Fig. 2 Electrical metric encoding particle lateral position. a Diagram showing the electrode design and the wiring scheme. Two differential current measurements are taken: a transverse measurement $\left(I_{\mathrm{TSV}}\right)$ and an oblique measurement $\left(I_{\mathrm{OBQ}}\right)$. b, c FEM simulations of the differential current signals (real part) generated by a flowing particle (6- $\mu \mathrm{m}$-diameter insulating bead). b When a particle travels near the stimulation electrodes (i.e. in the positive $x$ region, trajectory 1) the peak-to-peak distances $\Delta z_{\mathrm{TSV}}$ and $\Delta z_{\mathrm{OBQ}}$ are similar and their ratio is close to one. $\mathbf{c}$ When a particle travels near the measuring electrodes (i.e. in the negative $x$ region, trajectory 2) $\Delta z_{\mathrm{OBQ}}$ is greater than $\Delta z_{\mathrm{TSV}}$ and their ratio is greater than one. d Simulated peak-to-peak time ratio $R$ (equivalent to $\Delta z_{\mathrm{OBQ}} / \Delta z_{\mathrm{TSV}}$ ) versus particle $x$-coordinate. The one-to-one relationship between $R$ and $x$ shows that the ratio $R$ can be used to estimate particle lateral position 
Figure $2 \mathrm{~b}$ [resp. c] shows the trace (real part) obtained in simulation when a dielectric bead travels along trajectory 1 [resp. 2] depicted in Fig. 2a. The differential signals $I_{\mathrm{TSV}}$ and $I_{\mathrm{OBQ}}$ exhibit a bipolar profile, and the relevant peak-to-peak distances are denoted by $\Delta z_{\mathrm{TSV}}$ and $\Delta z_{\mathrm{OBQ}}$, respectively. While $\Delta z_{\mathrm{TSV}}$ is the same for both trajectories, $\Delta z_{\mathrm{OBQ}}$ depends on particle lateral position: it is higher for particles travelling near the sensing electrodes (trajectory 2, Fig. 2c) than for particles travelling near the stimulation electrodes (trajectory 1, Fig. 2b). From the experimental point of view, the peakto-peak times $\Delta t_{\mathrm{TSV}}$ and $\Delta t_{\mathrm{OBQ}}$ are available, instead of the peak-to-peak distances $\Delta z_{\mathrm{TSV}}$ and $\Delta z_{\mathrm{OBQ}}$. Although $\Delta t_{\mathrm{TSV}}$ and $\Delta t_{\mathrm{OBQ}}$ depend on particle velocity, their ratio

$R=\Delta t_{\mathrm{OBQ}} / \Delta t_{\mathrm{TSV}}$,

coinciding with $\Delta z_{\mathrm{OBQ}} / \Delta z_{\mathrm{TSV}}$, is a dimensionless quantity, independent of velocity, that correlates with particle lateral position. Consequently, $R$ can be used to obtain an electrical estimate $X$ of particle $x$-coordinate.

A simple geometric argument, assuming straight current paths (Fig. 2a), yields the following linear estimate:

$X_{\text {lin }}=W\left(\frac{3}{2}-R\right)$,

where $W$ denotes the channel width. Simulation results (Fig. 2d) confirm that such a simple model is valid, except for a slight saturation at extremal lateral positions. A refined model, involving quadratic and cubic corrections, is introduced to cover the whole position range:

$X=W\left(\frac{3}{2}-R\right)\left[1+b_{1}\left(\frac{3}{2}-R\right)+b_{2}\left(\frac{3}{2}-R\right)^{2}\right]$.

The calibration procedure of the parameters $b_{1}$ and $b_{2}$ is described in Sect. 4.3.

\subsection{Electrical estimate of particle vertical position and particle velocity}

In order to estimate particle vertical position (i.e. $y$-coordinate), the sensing zone comprising five electrodes spanning the main channel width (Fig. 1c) is used. The relevant wiring scheme, introduced in De Ninno et al. (2017), is depicted in Fig. 3a: an AC voltage signal is applied to the central electrode, and the difference in electric current flowing through the lateral electrodes is measured. Intermediate electrodes are left floating. This wiring scheme results in a non-homogeneous electric field distribution along the channel axis (z-direction), characterized by weak-field regions in front of the floating electrodes, which in turn are reflected as local minima in the collected current. Therefore, the resulting signal trace exhibits a bipolar double-Gaussian (BDG) profile and is denoted as $I_{\mathrm{BDG}}$. (a)
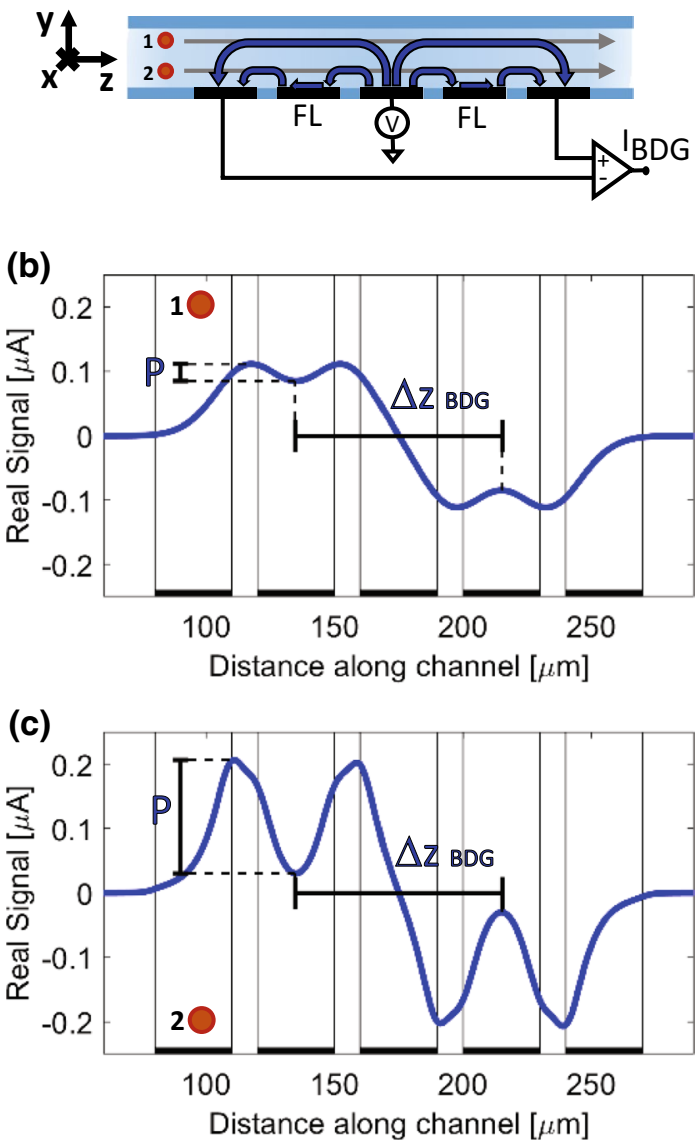

(d)

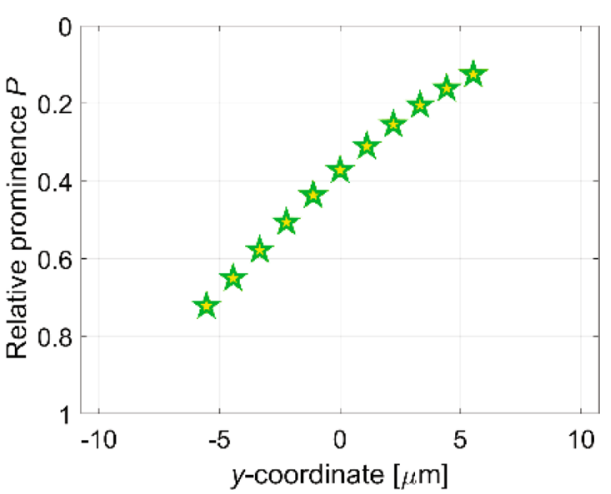

Fig. 3 Electrical metric encoding particle vertical position. a Diagram showing the electrode design and the wiring scheme. Compared to the standard three-electrode measurement configuration [e.g. Gawad et al. (2001)], two additional floating electrodes are inserted between the stimulation and the measuring electrodes. These electrodes create a non-homogeneous electric field distribution, shunting particles travelling close to them. b, c FEM simulations of the differential current signal (real part) generated by a flowing particle (6- $\mu \mathrm{m}$-diameter insulating bead). The signal exhibits bipolar double-Gaussian (BDG) shape which is characterized by the relative prominence $P$ of the peaks compared to the saddle. b When a particle travels far from the electrodes (i.e. in the positive $y$ region, trajectory 1 ) the relative prominence $P$ is low. $\mathbf{c}$ When a particle travels near the electrodes (i.e. in the negative $y$ region, trajectory 2) the relative prominence $P$ is high. d Simulated relative prominence $P$ versus particle $y$-coordinate. The one-to-one relationship between $P$ and $y$ shows that the relative prominence $P$ can be used to estimate particle vertical position 
Figure $3 \mathrm{~b}$ [resp. c] shows the trace (real part) obtained in simulation when a dielectric bead travels along trajectory 1 [resp. 2] depicted in Fig. 3a. The prominence of the two peaks with respect to the saddle in between is higher for particles travelling close to the electrodes (trajectory 2, Fig. 3c) than for particles travelling away from the electrodes (trajectory 1, Fig. 3b). Because signal amplitude also depends on particle size, the following normalized metric, referred to as relative prominence, was introduced in De Ninno et al. (2017):

$P=\frac{M-m}{M}$,

where $m$ and $M$ are the signal amplitude at the saddle and peaks, respectively.

Finite element simulations (Fig. 3d) show that the relative prominence $P$ correlates with the height of particle trajectory ( $y$-coordinate): the higher the former, the lower the latter. This claim has been experimentally supported in De Ninno et al. (2017) by means of a quantitative defocusing approach (Wu et al. 2005). As a consequence, the relative prominence $P$ can be used to obtain an electrical estimate $Y$ of particle $y$-coordinate. In particular, a quadratic model is used in this work:

$Y=H\left(c_{0}+c_{1} P+c_{2} P^{2}\right)$,

where $H$ denotes channel height. As shown in Caselli and Bisegna (2017), the parameters $c_{i}$ depend on the experimental setup (e.g. buffer conductivity, frequency of the AC stimulation, electrode double-layer capacitance). The relevant calibration procedure is described in Sect. 4.3.

An electrical estimate $V$ of particle velocity is obtained from the signal $I_{\mathrm{BDG}}$ as follows:

$V=\Delta z_{\mathrm{BDG}} / \Delta t_{\mathrm{BDG}}$,

where $\Delta z_{\mathrm{BDG}}$ is the distance between the centres of the floating electrodes ( $\Delta z_{\mathrm{BDG}}=80 \mu \mathrm{m}$ for the present geometry) and $\Delta t_{\mathrm{BDG}}$ is the relevant transit time (De Ninno et al. 2017).

\section{Experimental}

\subsection{Microfluidic chip}

The impedance chip consists of a glass microscope slide with integrated gold microelectrodes, bonded to a PDMSembedded microchannel (Fig. 1). The device was fabricated following standard microfabrication techniques, as reported elsewhere (De Ninno et al. 2017). The main channel was $40 \mu \mathrm{m}$ wide and $21.5 \mu \mathrm{m}$ high. The lateral channels in the lateral position determination region were $30 \mu \mathrm{m}$ wide and $30 \mu \mathrm{m}$ apart from each other, with electrodes recessed by $20 \mu \mathrm{m}$ with respect to the main channel. The five electrodes in the vertical position determination region, spanning the main channel width, were $30 \mu \mathrm{m}$ wide with a $10-\mu \mathrm{m}$ gap between them. For fluidic access, Teflon tubing (OD: 1/16") was inserted into chip inlet and connected to a 500- $\mu$ glass syringe (Hamilton) loaded on a syringe pump (Elite 11, Harvard Apparatus). The chip was connected electrically to a custom chip holder using pogo pins.

\subsection{Sample preparation}

The system was tested using polystyrene beads with diameters of 6 or $10 \mu \mathrm{m}$ (Sigma-Aldrich). The beads were resuspended to a concentration of approximately $10^{6}$ beads/ $\mathrm{ml}$ in PBS containing $0.1 \%$ Tween 20 and enough sucrose to match particle density $\left(1050 \mathrm{~kg} / \mathrm{m}^{3}\right)$. The conductivity of the final medium was $1.1 \mathrm{~S} / \mathrm{m}$. The samples were sonicated prior to the experiments, in order to prevent bead aggregation. Samples were pumped through the device at a flow rate of 3,10 or $30 \mu \mathrm{l} / \mathrm{min}$.

\subsection{Impedance data acquisition and processing}

Electrical signals were measured using two transimpedance amplifiers (HF2TA, Zurich Instruments) and an impedance spectroscope (HF2IS, Zurich Instruments). Details of the electrical connections are reported in Supplementary material, Figure S1. Excitation signals of $8 \mathrm{~V}, 1 \mathrm{MHz}$ and $6 \mathrm{~V}$, $884.8 \mathrm{kHz}$ were used for the determination of lateral and vertical positions, respectively. Differential currents were sampled at $115.2 \mathrm{kSa} / \mathrm{s}$, with 6,20 or $60 \mathrm{kHz}$ filter bandwidth, respectively, for 3,10 or $30 \mu \mathrm{l} / \mathrm{min}$ flow rate.

Event detection in the data streams was performed with a previously reported algorithm (Caselli and Bisegna 2016), and a simple MATLAB script was used for event feature extraction by template fitting (Spencer et al. 2016; De Ninno et al. 2017). In particular, for each detected event the ratio $R$ (Eq. 1) was computed from the peak-to-peak times of the oblique and transverse signals $I_{\mathrm{OBQ}}$ and $I_{\mathrm{TSV}}$, whereas the relative prominence $P$ (Eq. 4 ) and the transit time $\Delta t_{\mathrm{BDG}}$ were extracted from the bipolar double-Gaussian signal $I_{\mathrm{BDG}}$. Parameters $R, P$ and $\Delta t_{\mathrm{BDG}}$ were then used to compute electrical position $X$, electrical height $Y$ and electrical velocity $V$ by means of Eqs. (3), (5) and (6), respectively.

\subsection{Optical data acquisition and processing}

For validation purposes, independent estimates of particle cross-sectional position and velocity were obtained by means of an optical approach. The optical recording region was located downstream the electrical sensing region. Images of a $1024 \times 112$ pixels area around the channel were recorded using a high-speed camera (Photron FASTCAM Mini UX100, $4000 \mathrm{fps}, 4 \mu \mathrm{s}$ shutter time) mounted on an 
inverted microscope (Zeiss Axio Observer, 20× objective). Images were first saved on the camera local memory (10.6 GB) and then moved to a desktop computer. These settings allowed for a continuous optical recording of $25 \mathrm{~s}$.

In order to ease the image segmentation step (i.e. the identification of beads in the recorded frames), the electrical velocity $V$ of each event detected in the impedance data streams was used to predict particle entrance time in the optical region, thus reducing the computational cost of image processing. For each particle, the optical lateral position $X_{\mathrm{opt}}$ was computed as the distance between the particle centre and the channel $z$-axis (Fig. 4a), whereas the optical velocity $V_{\text {opt }}$ was computed as the product of the distance travelled by the particle across two consecutive frames and the camera frame rate (Fig. 4b). The optical vertical position $Y_{\text {opt }}$ (Fig. 4c) was obtained by using a quantitative defocusing approach (Wu et al. 2005; Winer et al. 2014) based on

(a) LATERAL POSITION
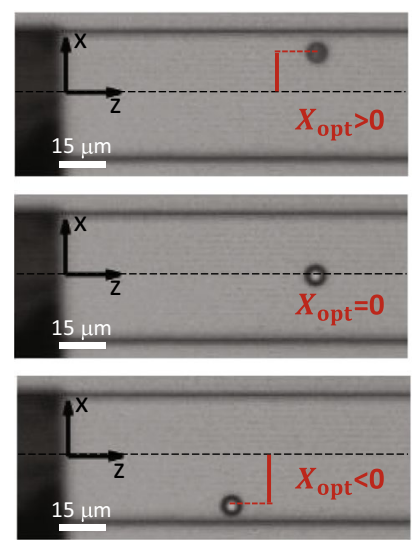

(b) VELOCITY $\quad V_{\text {opt }}=\frac{\Delta Z_{\text {opt }}}{\Delta t}$

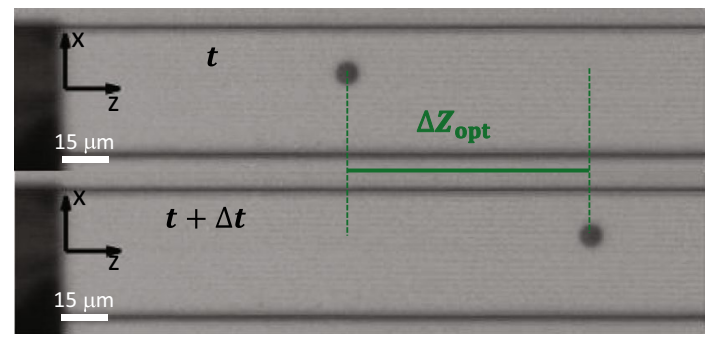

Fig. 4 Optical estimate of particle position and velocity. The optical recording region is located downstream the electrical sensing region. The dark area on the left in panels (a) and (b) is part of the rightmost electrode shown in Fig. 1a. a Lateral position $X_{\text {opt }}$ is determined by measuring the distance between particle centre and channel $z$-axis. $\mathbf{b}$ Velocity $V_{\text {opt }}$ is determined by measuring the distance travelled by a particle along the $z$-axis between two consecutive frames and multiplying it by the camera frame rate $(1 / \Delta t)$. c Vertical position $Y_{\mathrm{opt}}$ is determined by measuring particle central intensity and referring to a quantitative defocusing calibration set calibration data relating the $y$-position of a particle to its observed central intensity (De Ninno et al. 2017).

\subsection{Particle inertial focusing}

The onset of particle focusing can be observed in the laminar regime, when inertial effects start to become relevant. It can be predicted by particle Reynolds number, defined for a rectangular channel as (Di Carlo et al. 2007):

$R e_{\mathrm{p}}=\operatorname{Re}\left(\frac{a}{D_{h}}\right)^{2}=\frac{\rho U a^{2}}{\mu D_{h}}$

where $R e$ is channel Reynolds number, $a$ is particle diameter, $U$ is fluid maximum velocity, $D_{h}$ is cannel hydraulic diameter (defined as $2 W H /(W+H)$ ) and $\mu$ and $\rho$ are fluid dynamic viscosity and density, respectively.

At $R e_{\mathrm{p}} \geq 1$, randomly dispersed particles in a rectangular microchannel tend to order in specific equilibrium positions. They first migrate from the channel bulk towards equilibrium positions near the walls (fast migration), and then, they migrate parallel to channel walls into wall-centred equilibrium positions (slow migration) (Zhou and Papautsky 2013). In a square channel, there are four stable position in correspondence with the middle of each wall, whereas in a rectangular channel the positions close to the short walls become unstable and only two equilibrium position are left (Zhou and Papautsky 2013; Amini et al. 2014).

\section{Results}

\subsection{Information provided by the electrical metrics $R$ and $P$}

Figure 5 shows the density plot for 6 - $\mu$ m-diameter beads pumped at $3 \mu \mathrm{l} / \mathrm{min}$, with the relative prominence $P$ plotted against the peak-to-peak time ratio $R$. The particle Reynolds number $R e_{\mathrm{p}}$, equal to 0.11 , implies that little inertial focusing is established, so that particles adopt a random distribution in the rectangular channel cross section. Since a one-to-one monotonic decreasing relationship prevails between $R$ and $x$ [resp. $P$ and $y$ ], topological information on particle position in the cross section is provided by the $(R, P)$ density plot. In particular, particles are expected to visit a rectangular region, as experimentally confirmed.

Examples of experimental single-particle impedance signals are shown in Fig. 5, along with arrows indicating the position of the particle on the density plot. Particles (i), (ii), (iv) and (v) have trajectories near the corners of the cross section. As an example, particle (i) flows near the lowerleft channel corner, because its signal exhibits high relative prominence $P$ (i.e. low $y$-coordinate) and high peak-to-peak 

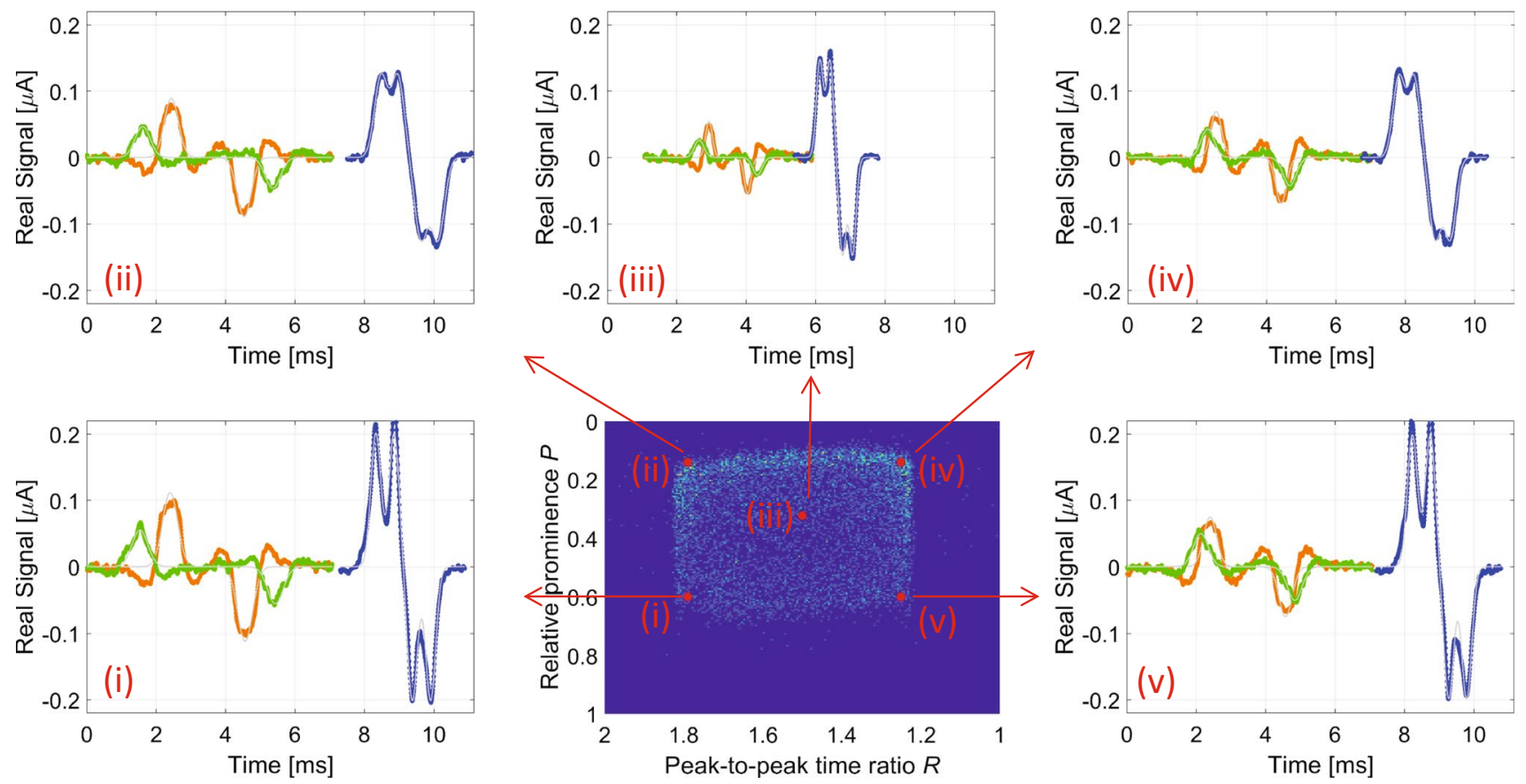

Fig. 5 Density plot for $6-\mu \mathrm{m}$-diameter beads (flow rate $3 \mu \mathrm{l} / \mathrm{min}$ ), with the relative prominence $P$ plotted against the peak-to-peak time ratio $R$. $\mathbf{i}-\mathbf{v}$ Are experimental single-particle signals for the data points in the density plot ( $I_{\mathrm{TSV}}$ : orange line; $I_{\mathrm{OBQ}}$ : green line; $I_{\mathrm{BDG}}$ : blue line).

time ratio $R$ (i.e. low $x$-coordinate). Particle (iii), exhibiting intermediate values of $R$ and $P$ and shortest $\Delta t_{\mathrm{BDG}}$ (i.e. highest velocity), flows along the channel axis.

\subsection{Inertial focusing detection}

The topological information provided by the electrical metrics $R$ and $P$, along with the electrical velocity $V$, was used to investigate inertial focusing effects. To this aim, different degrees of focusing were obtained experimentally by varying flow rate and beads diameter, thus varying $R e_{\mathrm{p}}$. In particular, $R e_{\mathrm{p}}$ values from $0.11(6-\mu \mathrm{m}$-diameter beads, $3 \mu \mathrm{l} / \mathrm{min})$ to $3.14(10-\mu \mathrm{m}$-diameter beads, $30 \mu \mathrm{l} / \mathrm{min})$ were considered (Fig. 6).

As shown in the $(R, P)$ plane, at $R e_{\mathrm{p}}=0.11$ particles were randomly distributed in the cross section (Fig. 6a). By increasing $R e_{\mathrm{p}}$, particles migrated away from the channel centre towards lines parallel to the four channel walls (Fig. 6, first column). The higher the particle Reynolds number $R e_{\mathrm{p}}$, the more pronounced the hydrodynamic focusing. This effect was analysed on the $(R, V)$ plane as well (Fig. 6, second column): at low $R e_{\mathrm{p}}$, the complete parabolic velocity profile could be observed, whereas when $R e_{\mathrm{p}}$ was increased, the high-velocities region (relevant to particles travelling close to the channel centre) became progressively less populated and visited by fewer particles. At $R e_{\mathrm{p}}=3.14$, the parabolic velocity profile
Fitting templates are also shown (grey lines). The histogram of the root-mean-square error of the fit, normalized by peak amplitude, is reported in Supplementary material, Figure S2 (colour figure online)

collapsed to the zones corresponding to velocities of the preferential positions parallel to channel walls. The same behaviour could be observed on the $(P, V)$ plane (Fig. 6 , third column).

At all values of $R e_{\mathrm{p}}$, the ranges of $R$ and $P$ values visited by the $10-\mu \mathrm{m}$-diameter beads were slightly narrower than those of the 6 - $\mu \mathrm{m}$-diameter beads, since a narrower cross section is available to the centres of the former particles, due to their larger size. Moreover, compared to $6-\mu \mathrm{m}$ beads, $10-\mu \mathrm{m}$ beads showed more pronounced focusing when operated under the same hydrodynamic conditions (i.e. same $R e_{\mathrm{p}}$ ). This might be explained considering that for $10-\mu \mathrm{m}$ beads the ratio of bead diameter to channel hydraulic diameter, $a / D_{h}$, is close to 0.4 and additional steric effects become relevant (Amini et al. 2014).

\subsection{Calibration procedure used to map the $(R, P)$-plane onto the $(X, Y)$-plane}

In order to transform the electrical metrics $(R$ and $P$ ) into electrical estimates $(X$ and $Y)$ of the Cartesian coordinates of particle centre ( $x$ and $y$ ), model Eqs. (3) and (5) were used. The relevant model parameters were calibrated using the experiment at $R e_{\mathrm{p}}=0.11(6-\mu \mathrm{m}$-diameter beads at $3 \mu$ $1 /$ min flow rate), where particles adopt a random distribution in the channel cross section (Fig. 6a-c). In particular, 


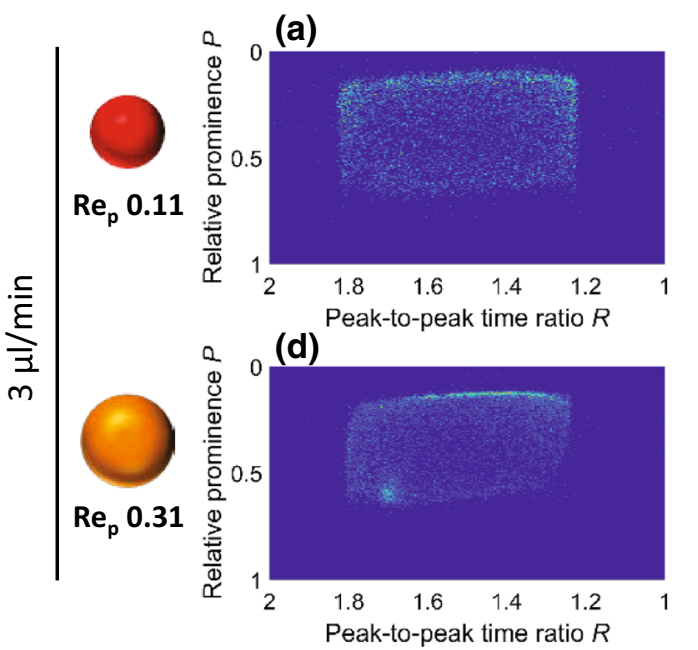

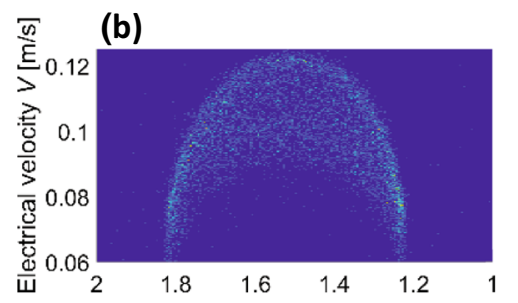
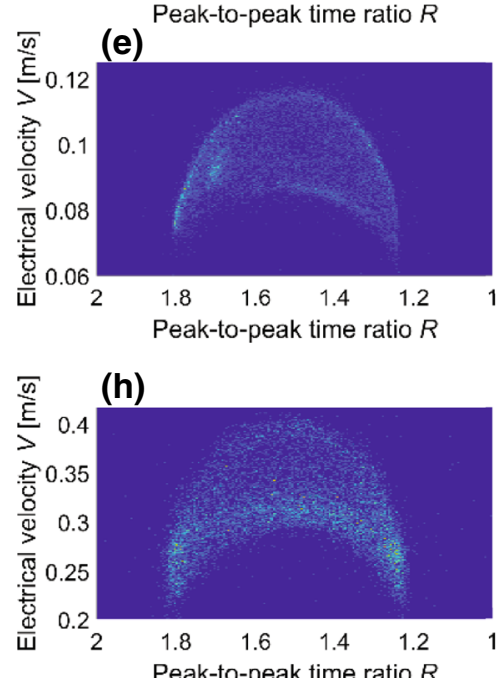

(k)
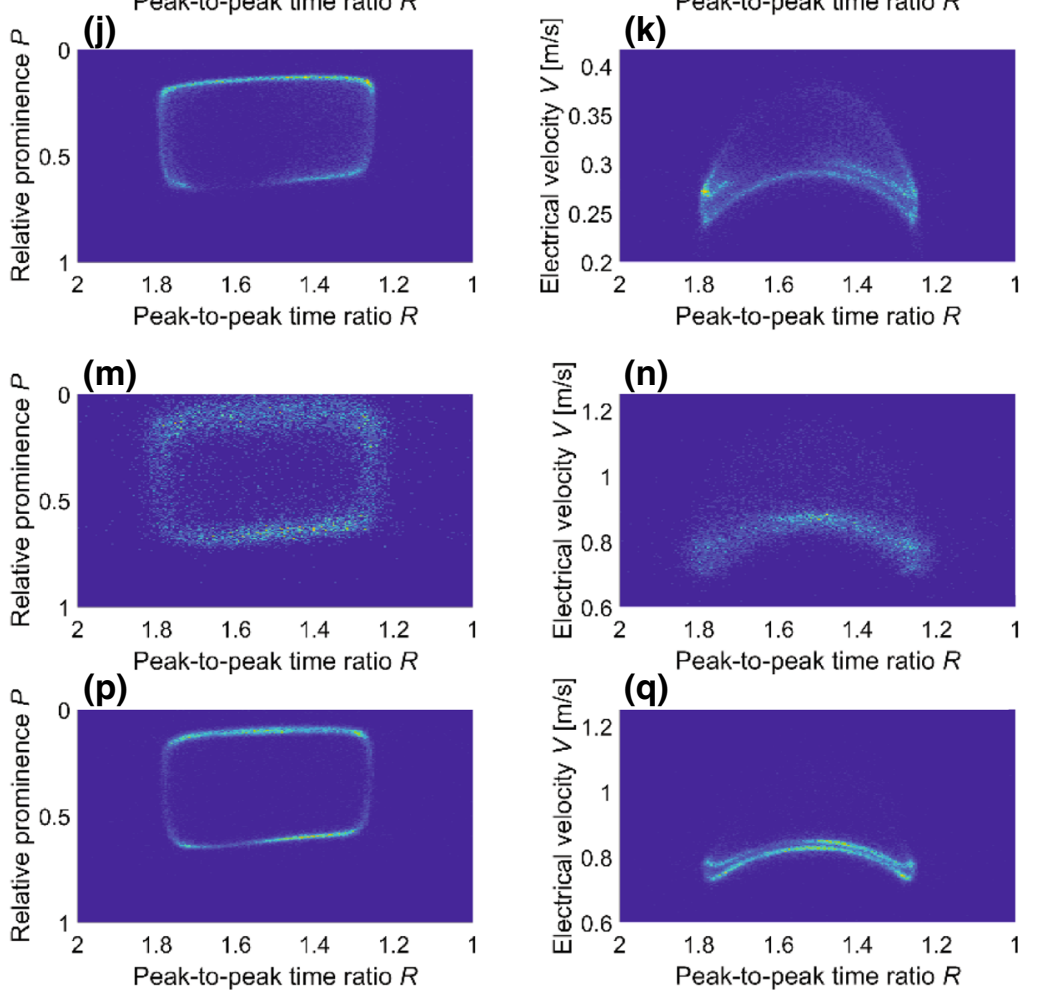
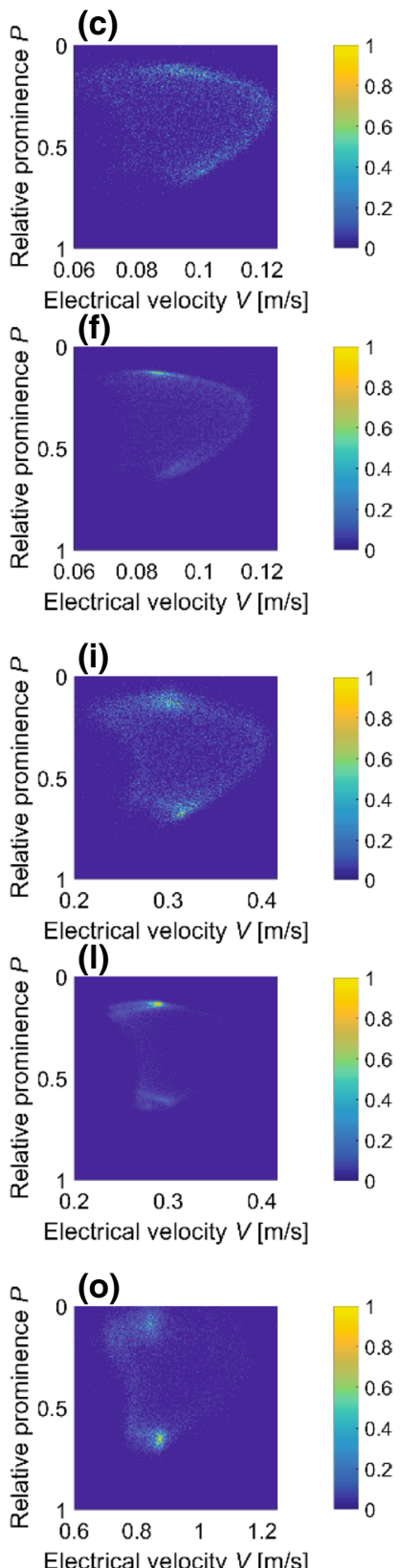

Electrical velocity $V[\mathrm{~m} / \mathrm{s}]$

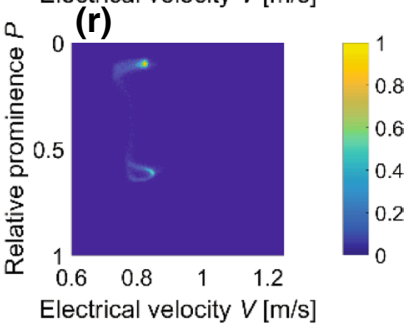

Fig. 6 Density plots of relative prominence $P$ versus peak-to-peak time ratio $R$ (first column); electrical velocity $V$ versus peak-to-peak time ratio $R$ (second column); electrical velocity $V$ versus relative prominence $P$ (third column). Six- $\mu$ m-diameter beads (odd rows) or $10-\mu \mathrm{m}$-diameter beads (even rows) were pumped through the device at flow rates of $\mathbf{a}-\mathbf{f} 3 \mu \mathrm{l} / \mathrm{min}, \mathbf{g}-\mathbf{l} 10 \mu \mathrm{l} / \mathrm{min}$, and $\mathbf{m}-\mathbf{r} 30 \mu \mathrm{l} / \mathrm{min}$. The higher the particle Reynolds number $R e_{\mathrm{p}}$, the more pronounced the hydrodynamic focusing. At least 6500 events are reported in each density plot and colour-bar values are normalized with respect to the highest bin count (colour figure online) 
accounting for finite particle diameter $a=6 \mu \mathrm{m}$, and allowing for a small gap, $g \approx 2 \mu \mathrm{m}$, between particle boundary and channel walls, particle centre coordinates satisfy the following inequalities:

$|x| \leq \frac{W}{2}-s, \quad|y| \leq \frac{H}{2}-s$,

where $s=(a / 2)+g$. Accordingly, parameters $b_{1}$ and $b_{2}$ appearing in Eq. (3) were determined by imposing the conditions:

$X\left(R_{\mathrm{L}}\right)=\frac{W}{2}-s, \quad X\left(R_{\mathrm{H}}\right)=-\frac{W}{2}+s$,

where $R_{\mathrm{L}}$ and $R_{\mathrm{H}}$ are the 1 and $99 \%$ percentile values of $R$, respectively (Fig. 7a, red lines). Their values are reported in Table 1 , along with the resulting calibration coefficients $b_{1}$ and $b_{2}$.

An analogous procedure was used to calibrate parameters $c_{0}, c_{1}$ and $c_{2}$ appearing in Eq. (5). However, an additional condition was needed in this case. Figure $7 \mathrm{~b}$ shows the density plot of the electrical velocity $V$ versus the relative prominence $P$. The curve $V_{\max }(P)$ that gives the maximum velocity $V$ measured at each relative prominence value $P$ is also plotted (green curve). It was computed using cubic $95 \%$ quantile regression instead of maxima search, in order to obtain a robust algorithm against outliers. Because, for any $y$, the maximum particle velocity in the channel cross section is attained at $x=0$, and a one-to-one correspondence between $y$ and $P$ prevails, the curve $V_{\max }(P)$ represents the velocity profile on the line $x=0$, as a function of $P$. That curve attains its maximum value at a relative prominence value $P_{\mathrm{M}}$ which corresponds to $y=0$, since the maximum velocity is attained at channel centre. (a)

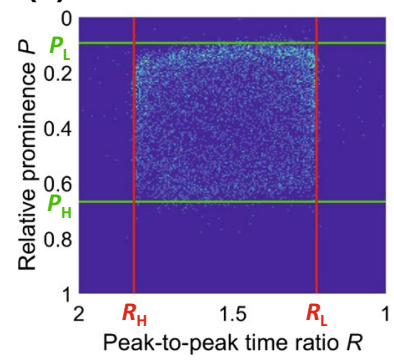

(b)

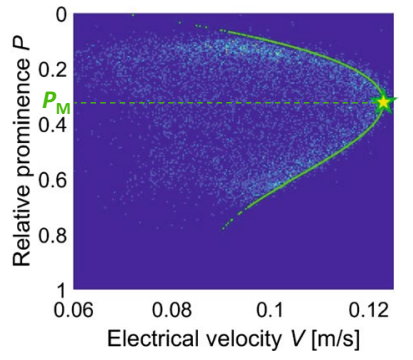

Fig. 7 Calibration experiment $(6-\mu \mathrm{m}$ beads, $3 \mu \mathrm{l} / \mathrm{min}$ flow rate, $\left.R e_{\mathrm{p}}=0.11\right)$. a Density plot of relative prominence $P$ versus peak-topeak time ratio $R$. The 1 and 99\% percentile values of $R$ (red lines) and $P$ (green lines) are shown. The corresponding values are labelled as $R_{\mathrm{L}}, R_{\mathrm{H}}$ (Table 1 ), and $P_{\mathrm{L}}, P_{\mathrm{H}}$ (Table 2). See also Supplementary material, Figure S3. b Density plot of electrical velocity $V$ versus relative prominence $P$. The cubic 95\% quantile regression is shown in green. The relative prominence value $P_{M}$ corresponding to the maximum velocity $V_{\max }=0.12 \mathrm{~m} / \mathrm{s}$ is visualized (Table 2). Colour-bar values are normalized with respect to the highest bin count (colour figure online)
Table 1 Values of 1 and $99 \%$ percentile of $R$ (Fig. 7a) and resulting calibration parameters (Eq. 3)

\begin{tabular}{llll}
\hline$R_{\mathrm{L}}$ & $R_{\mathrm{H}}$ & $b_{1}$ & $b_{2}$ \\
\hline 1.22 & 1.82 & 0.48 & 3.14
\end{tabular}

Therefore, the parameters $c_{0}, c_{1}, c_{2}$ entering Equation (5) were determined by imposing the conditions:

$Y\left(P_{\mathrm{L}}\right)=\frac{H}{2}-s, \quad Y\left(P_{\mathrm{H}}\right)=-\frac{H}{2}+s, \quad Y\left(P_{\mathrm{M}}\right)=0$,

where $P_{\mathrm{L}}$ and $P_{\mathrm{H}}$ are the 1 and $99 \%$ percentile values of $P$, respectively (Fig. 7a, green lines). The values of $P_{\mathrm{L}}, P_{\mathrm{M}}$ and $P_{\mathrm{H}}$ are reported in Table 2 , along with the resulting calibration coefficients $c_{0}, c_{1}$ and $c_{2}$.

\subsection{Optical validation of electrical position $(X, Y)$ and electrical velocity $V$}

The electrical estimates of particle lateral position $X$ (Eq. 3), vertical position $Y$ (Eq. 5) and velocity $V$ (Eq. 6) were compared with the optical estimates $X_{\mathrm{opt}}, Y_{\mathrm{opt}}$ and $V_{\mathrm{opt}}$ in Fig. 8 (6- $\mu \mathrm{m}$ beads, $10 \mu \mathrm{l} / \mathrm{min}$ flow rate).

The density plot of the electrical lateral position $X$ against the optical lateral position $X_{\text {opt }}$ shows a very good agreement between the two estimates along the whole position range (Fig. 8a, 0.97 correlation coefficient, $2.2 \mu \mathrm{m}$ rootmean-square difference, that is $5.6 \%$ of channel width). It is pointed out that even the very simple estimate $X_{\text {lin }}$ proposed in Eq. (2), based on a geometric argument and not requiring any calibration, provides a reasonably accurate estimate of particle lateral position as shown by the density plot of $X_{\text {lin }}$ versus $X_{\text {opt }}$ (Supplementary material, Figure S4).

A reasonable agreement is found between the electrical vertical position $Y$ and the optical one $Y_{\text {opt }}$ (Fig. 8b, 0.94 correlation coefficient, $1.7 \mu \mathrm{m}$ root-mean-square difference, that is $8.1 \%$ of channel height). As a matter of fact, the procedure used to estimate the lateral position $X_{\mathrm{opt}}$, which is based on bead segmentation within image frames, is more accurate than the procedure used to estimate the vertical position $Y_{\mathrm{opt}}$, which uses a calibration-based defocusing approach (De Ninno et al. 2017). Moreover, the electrical estimate of the vertical position $Y$, based on the relative prominence $P$, may exhibit some blurring due to fitting inaccuracy at low relative prominence values (corresponding to high $Y$ values). It is noticed that the electrical method used for the lateral

Table 2 Values of 1 and 99\% percentile of $P$ (Fig. 7a) and value of $P$ at maximum velocity (Fig. 7b). Resulting calibration parameters (Eq. 5)

\begin{tabular}{llllll}
\hline$P_{\mathrm{L}}$ & $P_{\mathrm{M}}$ & $P_{\mathrm{H}}$ & $c_{0}$ & $c_{1}$ & $c_{2}$ \\
\hline 0.09 & 0.32 & 0.67 & 0.40 & -1.44 & 0.67 \\
\hline
\end{tabular}


(a)

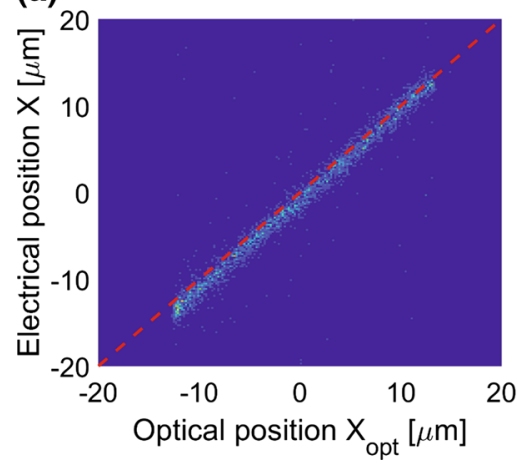

(b)

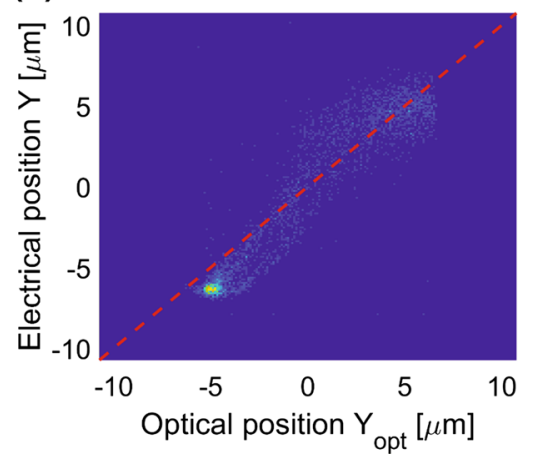

(c)

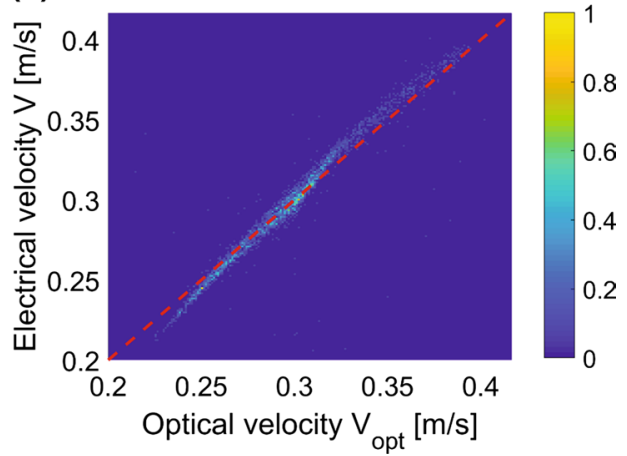

Fig. 8 Comparison between electrical estimates $X, Y, V$ and optical estimates $X_{\text {opt }}, Y_{\text {opt }}, V_{\text {opt }}(6-\mu \mathrm{m}$ beads, $10 \mu \mathrm{l} / \mathrm{min}$ flow rate). Data are shown as density plots (2500 events), and the bisector line is dashed in red. Colour-bar values are normalized with respect to the highest bin count. a Electrical lateral position $X$ (Eq. 3) versus optical lateral position $X_{\mathrm{opt}}(0.97$ correlation coefficient, $2.2 \mu \mathrm{m}$ root-mean-square

position determination could be replicated in a vertical configuration using five pairs of facing electrodes (Spencer et al. 2016), thus providing an even more accurate estimate of particle vertical position, at the expense of a more complex microfabrication process.

The density plot of the electrical velocity $V$ against the optical velocity $V_{\text {opt }}$ is shown in Fig. 8c. An excellent agreement is found between the two measurements ( 0.97 correlation coefficient, $0.01 \mathrm{~m} / \mathrm{s}$ root-mean-square difference, that is $2.3 \%$ of maximum particle velocity). The velocity range measured optically and electrically is $(0.20-0.39) \mathrm{m} / \mathrm{s}$, with average particle velocity of $0.30 \mathrm{~m} / \mathrm{s}$. By comparison, the velocity distribution in steady state, hydrodynamically fully developed, laminar flow for Newtonian fluids in rectangular channels (Spiga and Morini 1994) has maximum $0.39 \mathrm{~m} / \mathrm{s}$ and average $0.19 \mathrm{~m} / \mathrm{s}$ for the present channel cross section and flow rate. In fact, particles tend to avoid trajectories very close to the channel walls, so that their average velocity is greater than average fluid velocity.

\subsection{Quantitative particle positioning within the channel cross section}

The $R \mapsto X$ and $P \mapsto Y$ mappings (namely Eqs. 3 and 5 with the coefficient values reported in Tables 1 and 2) were applied to the data sets considered in Fig. 6. The resulting $(X, Y),(X, V)$ and $(Y, V)$ density plots are shown in Fig. 9. For each experimental condition, the percentage of particles falling in each bin of a $3 \times 3$ regular grid in the $(X, Y)$ plane (Fig. 9, first column) is also indicated (bin size $W / 3 \times H / 3$ ).

At $R e_{\mathrm{p}}=0.11$, when particles are randomly distributed, $21 \%$ of the particles visited the central bin and lower percentages were found in the other bins (especially the corner difference, that is $5.6 \%$ of channel width). b Electrical vertical position $Y$ (Eq. 5) versus optical vertical position $Y_{\text {opt }}(0.94$ correlation coefficient, $1.7 \mu \mathrm{m}$ root-mean-square difference, that is $8.1 \%$ of channel height). c Electrical velocity $V$ (Eq. 6) versus optical velocity $V_{\mathrm{opt}}$ (0.97 correlation coefficient, $0.01 \mathrm{~m} / \mathrm{s}$ root-mean-square difference, that is $2.3 \%$ of maximum particle velocity) (colour figure online)

bins), due to a minimum distance of particle centres from channel walls imposed by finite particle size. By increasing $R e_{\mathrm{p}}$, the particles moved away from the central bin towards peripheral bins. At $R e_{\mathrm{p}}=3.14$, only $1 \%$ of the particles visited the central bin, and the highest particle concentration was found in the bin corresponding to the centre of the long walls, in agreement with the literature (Zhou and Papautsky 2013; Amini et al. 2014) (see Supplementary material, Figure S5).

The evolution of particle distribution in the channel cross section as $R e_{\mathrm{p}}$ increases is reflected in changes of the velocity profiles observed in the $(X, V)$ and $(Y, V)$ planes (Fig. 9, second and third column, respectively).

\section{Conclusion}

In this work, we reported the first high-throughput electrical system for the measurement of cross-sectional position and velocity of single particles flowing through a microchannel. Lateral position was computed measuring the ratio of oblique and transverse transit times, whereas vertical position and velocity were extracted from the relative prominence and transit time of a bipolar double-Gaussian signal measured with a suited wiring scheme. Transit-time ratios and relative prominence values supplied valuable topological information on particle position in the microchannel cross section. Moreover, they could be transformed to quantitative estimates of lateral and vertical positions using a simple calibration procedure. Optical validation confirmed the soundness of the proposed approach. As an applicative example, the effects of particle size and flow rate on inertial particle focusing were investigated. 

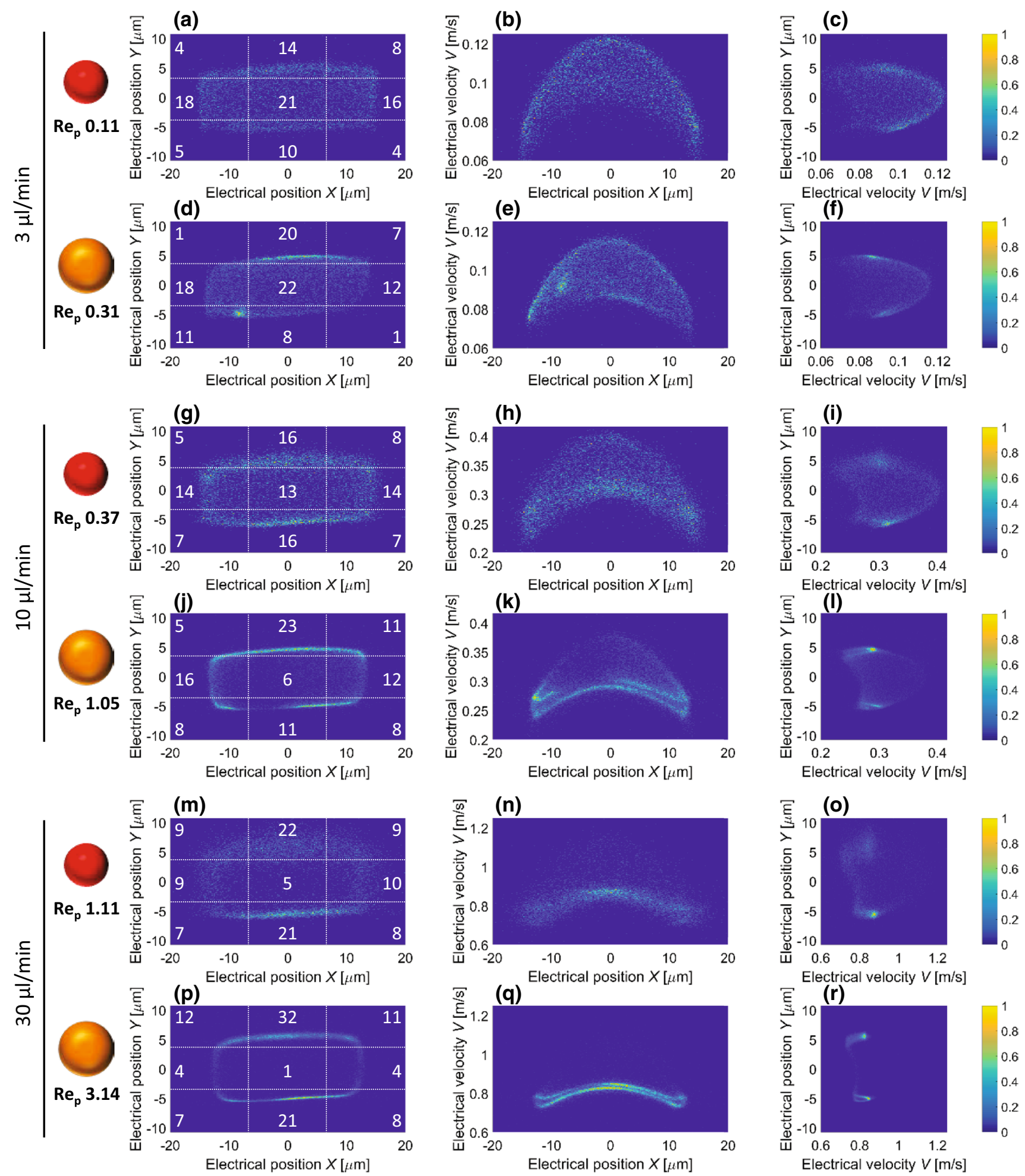

Fig. 9 Effect of particle Reynolds number $R e_{\mathrm{p}}$ on particle focusing. Measurements of $6-\mu \mathrm{m}$ (odd rows) or $10-\mu \mathrm{m}$-diameter (even rows) beads, pumped through the device at $3 \mu \mathrm{l} / \mathrm{min}(\mathbf{a}-\mathbf{f}), 10 \mu \mathrm{l} / \mathrm{min}(\mathbf{g}-\mathbf{l})$ or $30 \mu \mathrm{l} / \mathrm{min}(\mathbf{m}-\mathbf{r})$ flow rate, are shown. For each experimental condition, density plots of electrical position $Y$ versus electrical position $X$ (first column), electrical velocity $V$ versus electrical position $X$ (second column), electrical velocity $V$ versus electrical position $Y$ (third column) are reported. In the $(X, Y)$ density plot (first column), the percentage of particles falling in each bin of a $3 \times 3$ grid (white dotted lines) is also indicated. The higher the particle Reynolds number $R e_{\mathrm{p}}$, the more pronounced the hydrodynamic focusing. At least 6500 events are reported in each density plot and colour-bar values are normalized with respect to the highest bin count (colour figure online) 
The method has been demonstrated with reference to spherical beads. It applies as well to spherical cells, whose behaviour at low frequency (i.e. below the range of the $\beta$ -dispersion) is similar to that of an insulating particle (e.g. Schade-Kampmann et al. (2008), Haandbaek et al. (2014)). The method is also promising for nonspherical particles or cells because, although the latter yield impedance signals depending on their orientation (Jones 1995; Fernandez et al. 2017), the transit times and the relative prominence are not significantly affected by particle orientation.

This fully electrical label-free approach to particle monitoring, implemented with a simple coplanar chip layout and based on current signals instead of massive image data, represents an effective tool for investigating the behaviour of flowing particles at the microscale. Moreover, the device can be simultaneously used to perform single-cell impedance spectroscopy, thus achieving a multi-parametric characterization embracing size, membrane properties and intracellular conductivity in addition to particle position and velocity.

Acknowledgements This work was supported by the Scientific Independence of Young Researchers Programme (SIR 2014) under Grant RBSI14TX20-MUSIC "Multidimensional Single-Cell Microfluidic Impedance Cytometry".

Open Access This article is distributed under the terms of the Creative Commons Attribution 4.0 International License (http://creativeco mmons.org/licenses/by/4.0/), which permits unrestricted use, distribution, and reproduction in any medium, provided you give appropriate credit to the original author(s) and the source, provide a link to the Creative Commons license, and indicate if changes were made.

\section{References}

Amini H, Lee W, Di Carlo D (2014) Inertial microfluidic physics. Lab Chip 14:2739. https://doi.org/10.1039/C4LC00128A

Brazey B, Cottet J, Bolopion A, Van Lintel H, Renaud P, Gauthier M (2018) Impedance-based real-time position sensor for lab-on-achip devices. Lab Chip 18(5):818. https://doi.org/10.1039/C7LC0 1344B

Caselli F, Bisegna P (2016) A simple and robust event-detection algorithm for single-cell impedance cytometry. IEEE Trans Biomed Eng 63(2):415. https://doi.org/10.1109/TBME.2015.2462292

Caselli F, Bisegna P (2017) Simulation and performance analysis of a novel high-accuracy sheathless microfluidic impedance cytometer with coplanar electrode layout. Med Eng Phys 48:81. https://doi. org/10.1016/j.medengphy.2017.04.005

Caselli F, De Ninno A, Reale R, Businaro L, Bisegna P (2018) A novel wiring scheme for standard chips enabling high-accuracy impedance cytometry. Sens Actuators B Chem 256:580. https://doi. org/10.1016/j.snb.2017.10.113

Caselli F, Reale R, Nodargi NA, Bisegna P (2017) Numerical investigation of a novel wiring scheme enabling simple and accurate impedance cytometry. Micromachines 8(9):283. https://doi. org/10.3390/mi8090283

Caselli F, Reale R, De Ninno A, Errico V, Businaro L, Bisegna P (2017) 3D particle localization in a microfluidic impedance cytometer. In: 21 th international conference on miniaturized systems for chemistry and life sciences, MicroTAS

Cheung KC, Di Berardino M, Schade-Kampmann G, Hebeisen M, Pierzchalski A, Bocsi J, Mittag A, Tárnok A (2010) Microfluidic impedance-based flow cytometry. Cytom Part A 77(7):648. https ://doi.org/10.1002/cyto.a.20910

Cierpka C, Kähler CJ (2012) Particle imaging techniques for volumetric three-component (3D3C) velocity measurements in microfluidics. J Vis 15(1):1. https://doi.org/10.1007/s12650-011-0107-9

Di Carlo D (2009) Inertial microfluidics. Lab Chip 9:3038. https://doi. org/10.1039/B912547G

Di Carlo D, Irimia D, Tompkins RG, Toner M (2007) Continuous inertial focusing, ordering, and separation of particles in microchannels. Proc Natl Acad Sci USA 104:18892. https://doi.org/10.1073/ pnas.0704958104

De Ninno A, Errico V, Bertani FR, Businaro L, Bisegna P, Caselli F (2017) Coplanar electrode microfluidic chip enabling accurate sheathless impedance cytometry. Lab Chip 17:1158. https://doi. org/10.1039/C6LC01516F

Demierre N, Braschler T, Linderholm P, Seger U, van Lintel H, Renaud $P$ (2007) Characterization and optimization of liquid electrodes for lateral dielectrophoresis. Lab Chip 7:355. https://doi.org/10.1039/ B612866A

Esfandyarpour R, DiDonato MJ, Yang Y, Durmus NG, Harris JS, Davis RW (2017) Multifunctional, inexpensive, and reusable nanoparticle-printed biochip for cell manipulation and diagnosis. Proc Natl Acad Sci USA 114(8):E1306. https://doi.org/10.1073/ pnas. 1621318114

Fernandez RE, Rohani A, Farmehini V, Swami NS (2017) Review: microbial analysis in dielectrophoretic microfluidic systems. Anal Chim Acta 966:11. https://doi.org/10.1016/j.aca.2017.02.024

Gao Y, Magaud P, Baldas L, Lafforgue C, Abbas M, Colin S (2017) Self-ordered particle trains in inertial microchannel flows. Microfluid Nanofluid 21(10):154. https://doi.org/10.1007/s1040 4-017-1993-5

Gawad S, Schild L, Renaud P (2001) Micromachined impedance spectroscopy flow cytometer for cell analysis and particle sizing. Lab Chip 1(1):76. https://doi.org/10.1039/B103933B

Haandbaek N, Bürgel SC, Heer F, Hierlemann A (2014) Characterization of subcellular morphology of single yeast cells using high frequency microfluidic impedance cytometer. Lab Chip 14(2):369. https://doi.org/10.1039/C3LC50866H

Haandbæk N, Bürgel SC, Rudolf F, Heer F, Hierlemann A (2016) Characterization of single yeast cell phenotypes using microfluidic impedance cytometry and optical imaging. ACS Sens 1(8):1020. https://doi.org/10.1021/acssensors.6b00286

Jones TB (1995) Electromechanics of particles. Cambridge University Press, Cambridge

Lee SJ, Kim S (2009) Advanced particle-based velocimetry techniques for microscale flows. Microfluid Nanofluid 6(5):577. https://doi. org/10.1007/s10404-009-0409-6

Lin CH, Su SY (2016) Depth position detection for fast moving objects in sealed microchannel utilizing chromatic aberration. Biomicrofluidics 10(1):011904. https://doi.org/10.1063/1.4939943

McGrath JS, Honrado C, Spencer D, Horton B, Bridle HL, Morgan H (2017) Analysis of parasitic protozoa at the single-cell level using microfluidic impedance cytometry. Sci Rep 7:2601. https://doi. org/10.1038/s41598-017-02715-y

Mernier G, Duqi E, Renaud P (2012) Characterization of a novel impedance cytometer design and its integration with lateral focusing by dielectrophoresis. Lab Chip 12(21):4344. https://doi. org/10.1039/c2lc40551b

Muller PB, Rossi M, Marín AG, Barnkob R, Augustsson P, Laurell T, Kähler CJ, Bruus H (2013) Ultrasound-induced acoustophoretic motion of microparticles in three dimensions. Phys Rev E 88:023006. https://doi.org/10.1103/PhysRevE.88.023006 
Petchakup C, Li KHH, Hou HW (2017) Advances in single cell impedance cytometry for biomedical applications. Micromachines 8(3):87. https://doi.org/10.3390/mi8030087

Rohani A, Moore JH, Kashatus JA, Sesaki H, Kashatus DF, Swami NS (2017) Label-free quantification of intracellular mitochondrial dynamics using dielectrophoresis. Anal Chem 89(11):5757. https ://doi.org/10.1021/acs.analchem.6b04666

Rollo E, Tenaglia E, Genolet R, Bianchi E, Harari A, Coukos G, Guiducci C (2017) Label-free identification of activated T lymphocytes through tridimensional microsensors on chip. Biosens Bioelectron 94:193. https://doi.org/10.1016/j.bios.2017.02.047

Schade-Kampmann G, Huwiler A, Hebeisen M, Hessler T, Di Berardino M (2008) On-chip non-invasive and label-free cell discrimination by impedance spectroscopy. Cell Prolif 41(5):830. https:// doi.org/10.1111/j.1365-2184.2008.00548.x

Shaker M, Colella L, Caselli F, Bisegna P, Renaud P (2014) An impedance-based flow micro-cytometer for single cell morphology discrimination. Lab Chip 14(14):2548. https://doi.org/10.1039/c4lc0 $0221 \mathrm{k}$

Spencer D, Caselli F, Bisegna P, Morgan H (2016) High accuracy particle analysis using sheathless microfluidic impedance cytometry. Lab Chip 16(13):2467. https://doi.org/10.1039/c6lc00339g

Spencer D, Morgan H (2011) Positional dependence of particles in microfludic impedance cytometry. Lab Chip 11(7):1234. https:// doi.org/10.1039/c1lc20016j

Spiga M, Morini GL (1994) A symmetric solution for velocity profile in laminar flow through rectangular ducts. Int Commun Heat Mass Transf 21(4):469. https://doi.org/10.1016/0735-1933(94)90046-9
Sun T, Morgan H (2010) Single-cell microfluidic impedance cytometry: a review. Microfluid Nanofluid 8(4):423. https://doi. org/10.1007/s10404-010-0580-9

Tasadduq B, Wang G, Banani ME, Mao W, Lam W, Alexeev A, Sulchek T (2015) Three-dimensional particle tracking in microfluidic channel flow using in and out of focus diffraction. Flow Meas Instrum 45:218. https://doi.org/10.1016/j.flowmeasin st.2015.06.018

Wang H, Sobahi N, Han A (2017) Impedance spectroscopy-based cell/particle position detection in microfluidic systems. Lab Chip 17(7):1264. https://doi.org/10.1039/c6lc01223j

Williams SJ, Park C, Wereley ST (2010) Advances and applications on microfluidic velocimetry techniques. Microfluid Nanofluid 8(6):709. https://doi.org/10.1007/s10404-010-0588-1

Winer MH, Ahmadi A, Cheung KC (2014) Application of a threedimensional (3D) particle tracking method to microfluidic particle focusing. Lab Chip 14(8):1443. https://doi.org/10.1039/C3LC5 $1352 \mathrm{~A}$

Wu M, Roberts JW, Buckley M (2005) Three-dimensional fluorescent particle tracking at micron-scale using a single camera. Exp Fluids 38(4):461. https://doi.org/10.1007/s00348-004-0925-9

Wyatt Shields IV C, Reyes CD, Lopez GP (2015) Microfluidic cell sorting: a review of the advances in the separation of cells from debulking to rare cell isolation. Lab Chip 15(5):1230. https://doi. org/10.1039/C4LC01246A

Zhou J, Papautsky I (2013) Fundamentals of inertial focusing in microchannels. Lab Chip 13(6):1121. https://doi.org/10.1039/C2LC4 $1248 \mathrm{~A}$ 